\title{
Editorial
}

\section{Quantum Information and Entanglement}

\author{
Shao-Ming Fei, ${ }^{1}$ Sergio Albeverio, ${ }^{2}$ Adan Cabello, ${ }^{3}$ \\ Naihuan Jing, ${ }^{4}$ and Debashish Goswami ${ }^{5}$ \\ ${ }^{1}$ School of Mathematical Sciences, Capital Normal University, Beijing 100048, China \\ ${ }^{2}$ Institute of Applied Mathematics, University of Bonn, 53115 Bonn, Germany \\ ${ }^{3}$ Departamento de Fisica Aplicada II, Universidad de Sevilla, 41012 Sevilla, Spain \\ ${ }^{4}$ Department of Mathematics, North Carolina State University, Raleigh, NC 27695, USA \\ ${ }^{5}$ Statistics and Mathematics Unit, Indian Statistical Institute, Kolkata 700108, India
}

Correspondence should be addressed to Shao-Ming Fei, feishm@mail.cnu.edu.cn

Received 28 February 2010; Accepted 28 February 2010

Copyright (C) 2010 Shao-Ming Fei et al. This is an open access article distributed under the Creative Commons Attribution License, which permits unrestricted use, distribution, and reproduction in any medium, provided the original work is properly cited.

\section{Introduction}

As a rapidly expanding and cross-disciplinary subject, quantum information has attracted much attention and many leading theorists and experimentalists from physics, computer science, information, mathematics, as well as electronic engineering recently. Whereas the quantum entanglement, which can be traced back to the EPR paradox in 1935 and gave rise to the discussions on the foundations of quantum mechanics related to reality and locality, plays crucial roles in quantum information processing such as quantum computation, quantum teleportation, dense coding, quantum error correction, quantum cryptographic schemes, entanglement swapping, and remote states preparation. Many striking achievements have been witnessed for the past ten years.

The main focus of this special issue is on the theory of quantum entanglement and its applications to quantum information processing. As an international forum for researches to summarize the most recent developments and ideas, to present new results and at the same time to raise the awareness for such researches within the quantum information theory community in the field, the issue includes original research articles as well as review articles that will stimulate the continuing efforts in the quantum information processing, the related physicsm and the theory of quantum entanglement.

Among the quantum information processing quantum simulation was in fact the first proposed application for which quantum computers might give an exponential enhancement 
over classical computation, as was noted in 1982 by Feynman that simulating quantum dynamics on a classical computer was apparently intrinsically hard. On the other hand by the very definition of nonlocality, that is, violation of a Bell inequality, understanding the nonlocal correlations created upon measurement of some entangled quantum system is a problem that runs up against our common representation of the world. Bancal, Branciard, and Gisin propose a family of models that can simulate von Neumann measurements in the $x-y$ plane of the Bloch sphere on n-partite GHZ states using only bipartite nonlocal boxes. For the tripartite and fourpartite states, the models can be translated into classical communication schemes with finite average communication cost. Due to the interaction with the environment, quantum error correction is of significance in information processing. Guo, Zeng, and Lee investigate how to simplify the constructions of quantum error-correction codes via the quadratic residues. They present the quantum code that is constructed via the stabilizer formalism of an Abelian group and has advantages of being fast constructed with more efficiency than the previous quantum codes.

Quantum computing is the most important subject in quantum information processing. The operator quantum error correction encompasses active error correction and leads to improved threshold results in fault tolerant quantum computing. Qiao, Liu, and Ruda study the theory for a decoherence-free subspaces and subsystems in which quantum computing is performed in a decoherence-free subspace, although the total space still subject to a decoherence. A formalism for quantum computing in the decoherence-free subspaces is presented. The constructed subspaces are partial triangulated to the index related to the environment. The quantum states in the subspaces are just projected states which are ruled by a subdynamic kinetic equation. These projected states can be used to perform the ideal quantum logic operations without decoherence. Merkli, Berman, and Sigal describe their recent results on the resonant perturbation theory of decoherence and relaxation for quantum system with many qubits. The approach represents a rigorous analysis of the phenomenon of decoherence and relaxation for general $\mathrm{N}$-level systems coupled to reservoirs of the bosonic fields. The approach does not involve master equation approximations and applies to a wide variety of systems which are not explicitly solvable. The cluster state is a special, highly entangled quantum state that forms the universal resource for performing measurement-based quantum computation. Katz and Wang give a brief review of the theoretical foundations of cluster state quantum computation and propose a scheme for the generation of such entanglement in a solid-state medium with numerical calculations on the viability of the scheme for the creation of cluster states. Another scheme related to fault tolerant quantum computing is the topological one. Kádár, Marzuoli, and Rasetti study topological quantum field theories concerning this scheme.

Quantum dot is an important candidate for implementation of quantum information schemes. The theory of quantum dot has been extensively investigated. In Berthelot, Voisin, Delalande, Roussignol, Ferreira, and Cassabois's review, they present a general theoretical description of the extrinsic dephasing mechanism of spectral diffusion that dominates the decoherence dynamics in semiconductor quantum dots at low temperature. The limits of random telegraph and Gaussian stochastic noises are discussed. Seigneur, Gonzalez, Leuenberger, and Schoenfeld investigate the dynamics of entanglement between a quantum dot spin qubit and a single-photon qubit inside a quantum network node. The related robustness against various decoherence processes is also studied. Single photon and single photon-pair generation are fundamental in quantum information implementation. Kumano, Ekuni, Nakajima, Idutsu, Sasakura, and Suemune demonstrate the single-photon and polarization-correlated photon pair emission from a single semiconductor quantum dot. 
Quantum entanglement plays crucial roles in quantum information processing. Quantum entangled states have become the key ingredient in the rapidly expanding field of quantum information science. Li, Fei, and Li-Jost introduce some recent results in the theory of quantum entanglement: the separability criteria based on the Bloch representation, covariance matrix, normal form and entanglement witness; lower bounds and subadditivity property of concurrence and tangle; fully entangled fraction related to the optimal fidelity of quantum teleportation and entanglement distillation. Bayat and Bose study the possibility of using an uniformly coupled finite antiferromagnetic spin- $1 / 2$ Heisenberg chain as a channel for transmitting entanglement. One member of a pair of maximally entangled spins is initially appended to one end of a chain in its ground state and the dynamical propagation of this entanglement to the other end is calculated. It is shown that compared to the analogous scheme with a ferromagnetic chain in its ground state, in this scheme the entanglement is transmitted faster, with less decay, with a much higher purity. Moreover the nonzero temperatures and depolarizing environments are both found to be less destructive in comparison to the ferromagnetic case. Casado, Guerra, and Plácido apply the Wigner function formalism to partial Bell-state analysis using polarization entanglement produced in parametric down conversion. Two-photon statistics at a beam-splitter are reproduced by a wavelike description with zeropoint fluctuations of the electromagnetic field.

Quantum information processing is essentially based on quantum mechanics. Khrennikov discusses EPR-argument, one way quantum computing, and teleportation from the two versions of the projection postulate in quantum mechanics. Rocchi introduces a short survey on recent information theories and reviews some critical notes concerning the conceptualization of information in classical and quantum physics.

The Guest Editors of this special issue wish to thank all the authors who submitted manuscripts for consideration. Thanks also to the many individuals who served as referees of the submitted manuscripts.

Shao-Ming Fei Sergio Albeverio Adan Cabello Naihuan Jing Debashish Goswami 


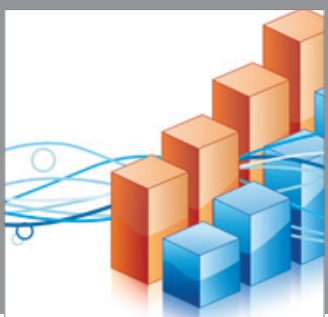

Advances in

Operations Research

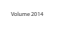

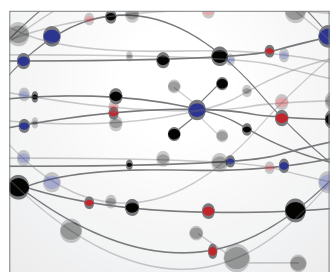

\section{The Scientific} World Journal
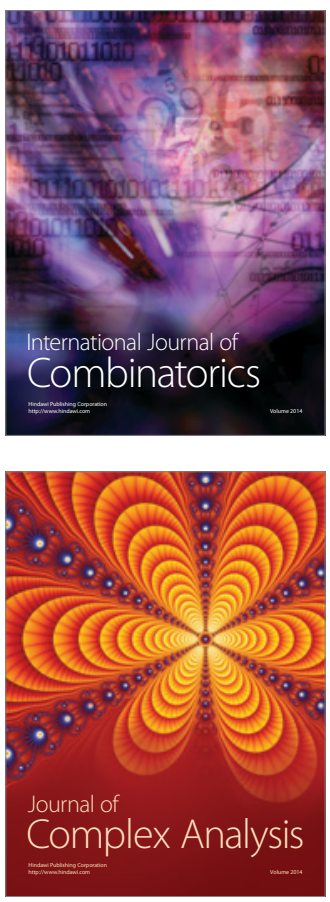

International Journal of

Mathematics and

Mathematical

Sciences
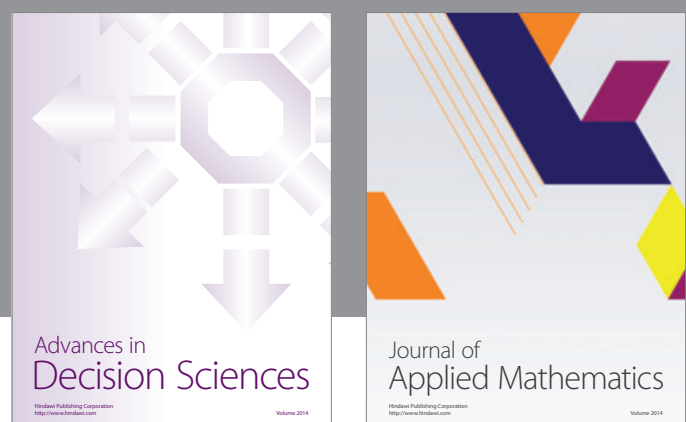

Journal of

Applied Mathematics
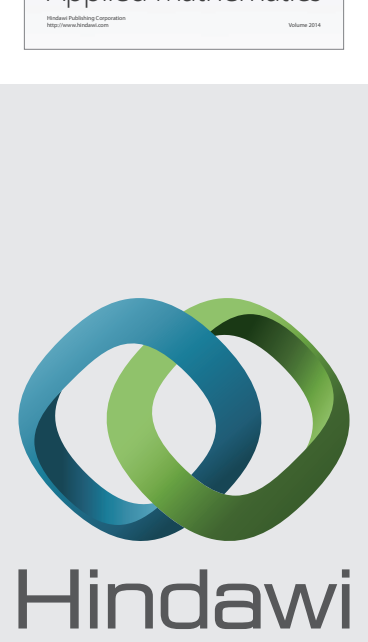

Submit your manuscripts at http://www.hindawi.com
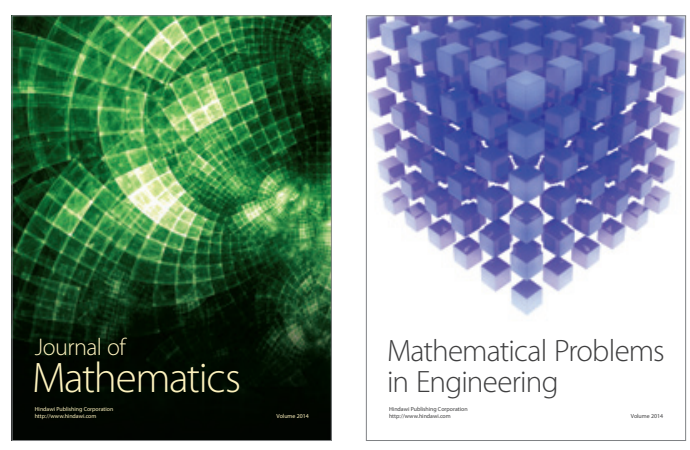

Mathematical Problems in Engineering
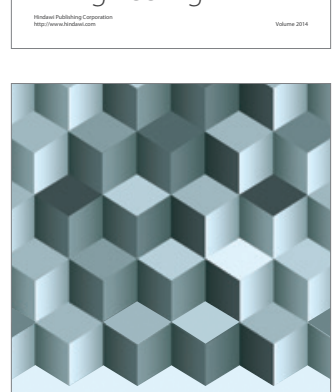

Journal of

Function Spaces
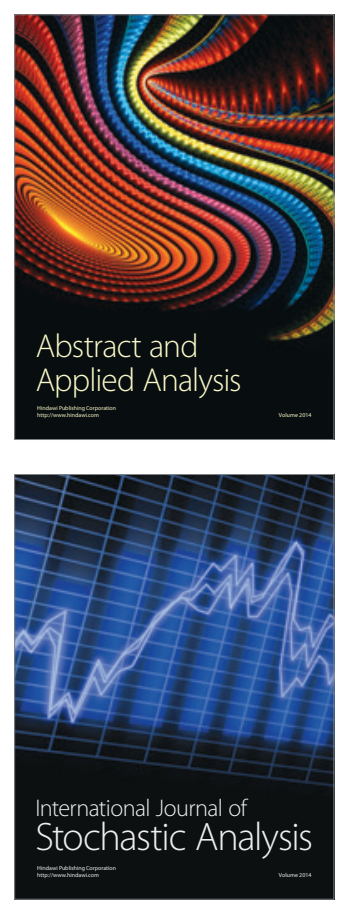

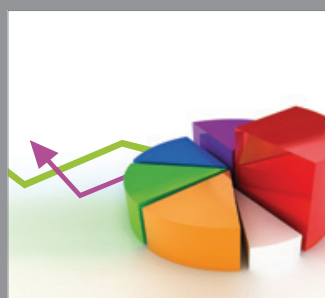

ournal of

Probability and Statistics

Promensencen
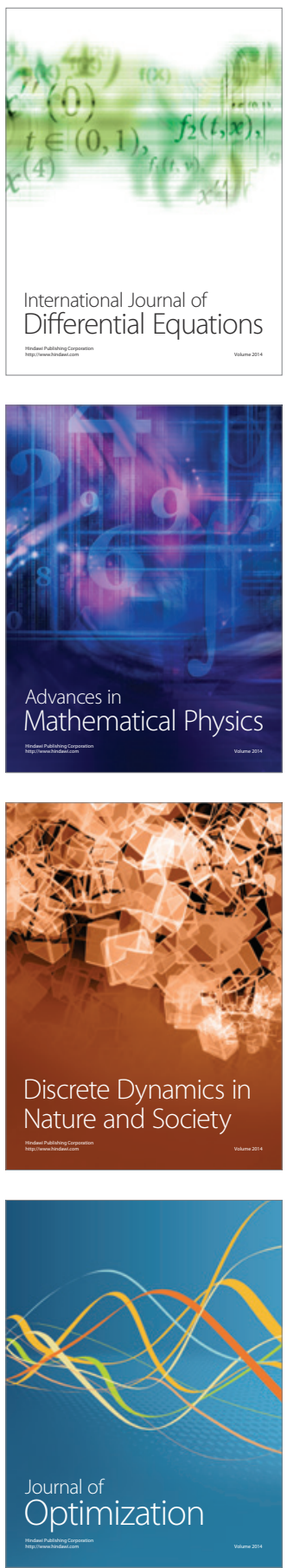